\title{
Adherence to the infant vitamin D supplementation policy in Ireland; data from two longitudinal, prospective birth cohort studies
}

\begin{abstract}
The high prevalence of maternal deficiency and the low vitamin D content of breastmilk places newborns and infants at particular risk of vitamin D deficiency. In response to an increase in the incidence of nutritional rickets, the Food Safety Authority of Ireland published an interim infant vitamin D supplementation policy in 2007, which was implemented by the Health Service Executive in Ireland in May 2010. This recommends that all infants be given a $5 \mu \mathrm{g}$ exclusive vitamin $\mathrm{D}_{3}$ supplement daily from birth to 12 months. As adherence is not monitored nationally and the policy has not been evaluated, the aim of this study was to conduct a detailed evaluation of supplementation practices across two maternal-infant cohort studies. Data from the prospective BASELINE (recruited 20082011) and COMBINE (recruited 2015-2017) birth cohorts, based in Cork, Ireland, were used to examine supplementation practices. After supplementation policy implementation, BASELINE collected vitamin D supplement use data $(n=1528)$ at 2,6 and 12 months. In COMBINE, 7 study visits from birth to 12 months allowed continuous collection of detailed longitudinal supplementation data in 364 participants. Use of supplemental vitamin D was higher in COMBINE than BASELINE at 2 (93 vs. $49 \%), 6(89$ vs. $64 \%)$ and 12 (72 vs. $44 \%$ ) months (all $P<0.001$ ). In COMBINE, $92 \%$ initiated vitamin D supplementation at birth and the median supplementation duration was $51(40,52)$ weeks, although there was a wide range $\left(3-52\right.$ weeks). $94 \%$ of COMBINE parents used a vitamin $\mathrm{D}_{3}$ only supplement and $88 \%$ used the recommended $5 \mu \mathrm{g}$ dose. Half (51\%) always supplemented daily; a further 33\% supplemented at least 36 times/week. Full policy adherence was defined as the provision of a $5 \mu$ g vitamin $\mathrm{D}_{3}$ supplement daily from birth; $64 \%$ adhered fully to 2 months and $52 \%$ did so to 6 months. By 12 months, $30 \%$ had adhered fully to the policy and a further $16 \%$ gave $5 \mu \mathrm{g}$ frequently for the full 12 months. This data indicates a high level of awareness of the supplementation policy amongst new mothers, with substantially higher rates of supplementation in our current cohort compared with BASELINE. While most parents gave an exclusive $5 \mu \mathrm{g}$ vitamin $\mathrm{D}_{3}$ supplement, frequency and duration were the key barriers to full policy adherence. Given the lack of a maternal vitamin $\mathrm{D}$ supplementation policy in Ireland and high prevalence of low vitamin D status at birth, supplementation of infants with vitamin D remains a vital public health policy.
\end{abstract}

\section{Conflict of Interest}

There is no conflict of interest 